\title{
Exclusive breastfeeding lowers the odds of childhood diarrhea and other medical conditions: evidence from the 2016 Ethiopian demographic and health survey
}

Tesfahun Mulatu', Nigus Bililign Yimer ${ }^{2}$, Birhan Alemnew ${ }^{3}$, Melese Linger ${ }^{1}$ and Misgan Legesse Liben ${ }^{* *}$ (D)

\begin{abstract}
Background: Lack of exclusive breastfeeding during the first 6 months of infant life contributes to childhood morbidity and mortality. This study aimed to investigate the association of exclusive breastfeeding and childhood illnesses in Ethiopia.

Methods: A secondary data analysis was conducted using data from the 2016 Ethiopian Demographic and Health Survey (EDHS). Descriptive and multivariable logistic regression analyses were carried out.

Results: A total of 1034 mother-infant pairs were included in the analysis. The overall magnitude of exclusive breastfeeding among infants aged under 6 months was $87.6 \%$ (95\% Cl: 84.3-90.3\%). Compared to infants who were non-exclusively breastfed, the odds of having an illness with fever in the last 2 weeks among infants who were exclusively breastfed decreased by $66 \%$ (AOR: $0.34 ; 95 \% \mathrm{Cl}: 0.16,0.75$ ). Similarly, exclusively breastfed infants had lower odds of having an illness with a cough (AOR: 0.38; Cl: 0.20, 0.72) and having diarrhea (AOR: 0.33; Cl: 0.13, 0.83) compared to non-exclusively breastfed infants.
\end{abstract}

Conclusion: Exclusive breastfeeding lowers the odds of an illness with fever, illness with cough and diarrhea. The findings of this study implicate the need for promotion of exclusive breastfeeding in the country.

Keywords: Cough, Breastfeeding, Diarrhea, Fever, Sub-Saharan Africa

\section{Background}

Breast milk provides ideal nutrition to meet the infant's needs for growth and development. It protects against many infections, and may prevent some infant deaths. Hence, breastfeeding has well established short and long-term benefits in childhood. It reduces morbidity, mortality and the risk of hospitalization from diarrhea and respiratory tract infections [1].

\footnotetext{
* Correspondence: Imisganl@gmail.com

'School of Public Health, College of Health Science, Woldia University, P.o.box: 400, Woldia University, Weldiya, Amhara, Ethiopia

Full list of author information is available at the end of the article
}

Exclusive breastfeeding is an infant's consumption of only breast milk in the first 6 months of life. The World Health Organization (WHO) recommend that 95\% of children younger than 1 month and $90 \%$ of those younger than 6 months should be exclusively breastfed, and $90 \%$ of those aged 6-23 months should be partly breastfed. However, in low and middleincome countries, only $37 \%$ of children younger than 6 months of age are exclusively breastfed [2]. In Ethiopia, about $77 \%$ of infants scored low and medium breastfeeding performance index during the first 5 months of life [3], and only $58 \%$ of infants under age 6 months are exclusively breastfed [4]. 
Breastfeeding provides all the nutrients and water that a baby needs to grow and develop in the first 6 months $[5,6]$. Exclusive breastfeeding has well-established shortterm and long term benefits, particularly in the reduction of childhood morbidity and mortality due to diarrhea and respiratory infection [7]. Lower breastfeeding index has been associated with increased risks of diarrhea and fever [3]. Furthermore, an estimated 1.3 million lives would be saved worldwide by promoting exclusive breastfeeding [1].

The government of Ethiopia has adapted infant and young child feeding guidelines since 2004 [8] and the national nutrition program in 2013 [9] to unlock the lifesaving potential of optimal breastfeeding practices. The guideline is based on WHO recommendations that give emphasis on exclusive breastfeeding. Furthermore, the health extension program in Ethiopia aims at improving proper infant and young child nutrition, for instance, promotion of exclusive breastfeeding [8].

Nongovernmental organizations are also addressing the issue of exclusive breastfeeding in different regions of the country through advocacy, community mobilization, and mass communication [9]. However, little is known about the impact of different breastfeeding practices on childhood illnesses in Ethiopia. Therefore, this study aimed to investigate the association of exclusive breastfeeding and childhood illnesses in Ethiopia.

\section{Methods}

\section{Study setting and design}

The 2016 EDHS was designed to provide up-to-date estimates of key demographic and health indicators in Ethiopia. A detailed description of the study design and methodology of the 2016 is found elsewhere [8]. In brief, a stratified two-stage random sampling design was used to collect data from a nationally representative sample. In the first stage, a total of 645 Enumeration Areas (EA) (202 in urban areas and 443 in rural areas) were selected with probability proportional to EA size and with independent selection in each sampling stratum. In the second stage, a fixed number of 28 households per cluster were selected with an equal probability systematic selection from the newly created household listing. A total of 18,008 households were selected, of which 17,067 were occupied and 16,650 were successfully interviewed, thus yielding a household response rate of $98 \%$. In the selected households, the response rate among women age 15-49 was $94.6 \%$. The sampling frame for the current study consisted of 1036 infants last-born infants aged less than 6 months, currently living with their mothers. We excluded women with missing data on the question related to the outcomes of interest, exclusive breastfeeding practices and other covariates adjusted in the multivariable model. The final analytic sample consisted of 1034 participants.

\section{Outcome of interest}

The outcome of interest was childhood illnesses. Three childhood illnesses namely: illness with fever, diarrhea and illness with cough were assessed by asking the mother to recall if the infant had any of these illnesses in the last 2 weeks preceding the survey. Each of these variables was dummy coded to reflect the presence or absence of these illnesses. Women who responded "Don't know" were excluded.

\section{Exposure assessment}

Exclusive breastfeeding was defined based on mother's recall on feeds given to the infant on the previous day. Specifically, mothers were asked whether or not infants received any solid, semi-solid or soft foods $24 \mathrm{~h}$ preceding the survey.

\section{Covariates}

Based on existing literature, the following covariates were selected: age of mother at infant's birth, household wealth index, educational level, place of delivery, place of residence, region of residence, antenatal care (ANC checkup), mode of delivery, age of infant, sex of infant, birth order, vaccination status, source of drinking water, wealth index and toilet facility.

\section{Statistical analysis}

Data were analyzed using SPSS version 23 (IBM Corp. Released 2015. IBM SPSS Statistics for Windows, Version 23.0. Armonk, NY: IBM Corp.). Descriptive statistics and cross-tabulation were performed to describe the study variables. Bivariate associations between each covariate and exclusive breastfeeding were first examined using Rao-Scott chi-square test. Univariable and multivariable logistic regression models were employed to determine the association between exclusive breastfeeding and childhood illness. Variance inflation factor (VIF) was used to assess the presence of collinearity. Using a conservative threshold VIF value of 4, no co-linearity was detected. Crude odds ratios (COR) and adjusted odds ratios (AOR) were presented with 95\% confidence intervals. Each covariate were included in the multivariable model regardless of their statistical significance in the univariable analysis. In the final multivariable logistic regression model, the association of EBF with childhood illness was declared statistically significant at $p$ value < 0.05. As recommended by the DHS Program, sample weights that account for complex survey design and unequal probabilities of selection were incorporated in all analyses [10]. 


\section{Ethical issues}

The data were downloaded once approval was obtained from Measure DHS. The original DHS data were collected in confirmation with international and national ethical guidelines. The 2016 EDHS protocol was reviewed and approved by the Federal Democratic Republic of Ethiopia Ministry of Science and Technology and the Institutional Review Board of ICF International.

\section{Results}

Table 1 displays characteristics of the study sample. Overall, the prevalence of exclusive breastfeeding among infants aged under 6 months was 87.6\% (95\% CI: 84.390.3\%). The prevalence of illness with cough in under-6 month infants was 17.9\% (95\% CI: 14.7-21.7\%). About $12 \%$ of infants $(11.8 \%$; $95 \%$ CI: $9.4-14.8 \%)$ had illness with fever and 7.4\% (95\% CI: 5.4-10.1\%) had diarrhea in the last 2 weeks. The mean $( \pm \mathrm{SE})$ age of the mothers and their under six infants were $27.57(0.31)$ years and $2.50(0.08)$ months respectively. Majority $(71.5 \%)$ of the

Table 1 Characteristics of the study sample by exclusive breastfeeding status in Ethiopia, EDHS 2016 ( $n=1034)$

\begin{tabular}{|c|c|c|c|c|}
\hline \multirow[t]{2}{*}{ Variables } & \multirow[b]{2}{*}{$\begin{array}{l}\text { Overall } \\
\text { n (wt.\%) }\end{array}$} & \multicolumn{2}{|c|}{ Exclusive BF } & \multirow[b]{2}{*}{$p$} \\
\hline & & $\begin{array}{l}\text { No } \\
\text { n (wt.\%) }\end{array}$ & $\begin{array}{l}\text { Yes } \\
\text { n (wt.\%) }\end{array}$ & \\
\hline \multicolumn{5}{|l|}{ Maternal age } \\
\hline$<20$ & $143(13.3)$ & $18(9.4)$ & $125(13.9)$ & \multirow[t]{3}{*}{0.327} \\
\hline $20-34$ & 753 (71.5) & 75 (69.3) & 678 (71.8) & \\
\hline $35+$ & $138(15.2)$ & $17(21.3)$ & $121(14.4)$ & \\
\hline \multicolumn{5}{|l|}{ Wealth Index } \\
\hline Poorest & $394(24.4)$ & $39(17.5)$ & $355(25.4)$ & \multirow[t]{5}{*}{0.057} \\
\hline Poorer & $164(23.4)$ & $20(25.6)$ & $144(23.1)$ & \\
\hline Middle & $123(18.8)$ & $17(25.2)$ & $106(17.9)$ & \\
\hline Richer & $138(18.1)$ & $19(26.4)$ & $119(17.0)$ & \\
\hline Richest & $215(15.2)$ & $15(5.2)$ & $200(16.6)$ & \\
\hline \multicolumn{5}{|c|}{ Place of residence } \\
\hline Urban & $203(11.3)$ & $16(7.7)$ & $187(11.8)$ & \multirow[t]{2}{*}{0.265} \\
\hline Rural & $831(88.7)$ & $94(92.3)$ & 737 (88.2) & \\
\hline \multicolumn{5}{|c|}{ Education level of mothers } \\
\hline No education & 606 (59.4) & 73 (63.6) & $533(58.8)$ & \multirow[t]{4}{*}{0.797} \\
\hline Primary & $288(30.4)$ & $27(25.2)$ & 261 (31.2) & \\
\hline Secondary & $103(8.0)$ & $7(9.4)$ & $96(7.8)$ & \\
\hline Higher & $37(2.2)$ & $3(1.8)$ & $34(2.3)$ & \\
\hline \multicolumn{5}{|l|}{ Birth order } \\
\hline 1 & $216(22.1)$ & $21(16.5)$ & 195 (22.9) & \multirow[t]{4}{*}{0.098} \\
\hline $2-3$ & $318(28.2)$ & $38(25.5)$ & 280 (28.6) & \\
\hline $4-6$ & 328 (30.9) & $27(27.0)$ & 301 (31.5) & \\
\hline$\geq 7$ & 172 (18.7) & $24(30.9)$ & $148(17.0)$ & \\
\hline
\end{tabular}

Abbreviations: Wt: weighted. BF: Breastfeeding mothers were in the age group of 20 to 34 years. About 8 out of 9 mothers $(88.7 \%)$ were rural residents, and majority of them $(59.4 \%)$ had no formal education. Women who were in the poorest and poor wealth index were more likely to exclusively breastfeed (Table 1 ).

Only $8.8 \%$ of the households included in this analysis had improved toilet facility and nearly half of the households (54.1\%) had improved drinking water source. From those who were non- exclusively breastfed about $87 \%$ of them had unimproved toilet facility or had no toilet facility (open defecation) at all. One third of the mothers (31.3\%) had attended 4 or more ANC visits. Half of the mothers with non-exclusively breastfed neonates had no ANC visit and three forth of them had delivered at home which was significantly different ( $p$ value 0.037$)$ from those mothers with exclusively breastfed neonates. Nearly all of the mothers had vaginal delivery (97\%). Majority (73.0\%) of the mothers initiated breastfeeding within $1 \mathrm{~h}$ of birth and about 7 out of 10 mothers had given anything other than breast milk. About 9 out of 10 (91.4\%) under six infants were bottle-fed and there was significant difference $(p$ value $<0.001)$ in bottle feeding status between exclusively and non-exclusively breastfed neonates (Table 2).

Table 3 presents results from the univariable and multivariable logistic regression analyses. Exclusive breastfeeding was significantly associated with illness with fever, illness with cough and diarrhea. The odds of developing illness with fever in those infants exclusively breastfed were by 66\% (AOR: 0.34 (CI: $0.16,0.75)$ ) lower than those who did not fed exclusively. Those infants who were exclusively breastfeeding had lower odds of developing cough and diarrhea by $62 \%$ (AOR: 0.38 (CI: $0.20,0.72)$ ) and 67\% (AOR: 0.33 (CI: 0.13, 0.83)) respectively as compared to those who did not fed exclusively (Table 3).

\section{Discussion}

In the current study, the overall magnitude of exclusive breastfeeding among infants aged less than 6 months was $87.6 \%$ and exclusive breastfeeding (EBF) was significantly associated with decreased odds of childhood illnesses. EBF lowered the odds of illness with fever, illness with cough and diarrhea in the last 2 weeks. These associations were independent of potential confounders. Our findings adds to the extant literature by being the first to examine the association between exclusive breastfeeding and childhood illnesses in Ethiopia.

The present study showed that exclusive breastfeeding decreased the odds of illness with fever by $66 \%$. Exclusive breastfeeding is a strong predictor for infant survival in Ethiopia [11]. Evidence from a Scottish birth cohort reported formula-fed infants had increased rate of hospitalization for gastrointestinal and respiratory tract 
Table 2 Characteristics of mother-infant pairs in Ethiopia, EDHS $2016(n=1034)$

\begin{tabular}{|c|c|c|c|c|}
\hline \multirow[t]{2}{*}{ Variables } & \multirow[b]{2}{*}{$\begin{array}{l}\text { Overall } \\
\text { n (\%) }\end{array}$} & \multicolumn{2}{|c|}{ Exclusive BF } & \multirow[t]{2}{*}{$p$} \\
\hline & & $\begin{array}{l}\text { No } \\
\text { n (wt.\%) }\end{array}$ & $\begin{array}{l}\text { Yes } \\
\text { n (wt.\%) }\end{array}$ & \\
\hline \multicolumn{5}{|l|}{ Sex of infant } \\
\hline Male & $512(49.2)$ & $59(54.7)$ & $453(48.4)$ & \multirow[t]{2}{*}{0.356} \\
\hline Female & $522(50.8)$ & $51(45.3)$ & $471(51.6)$ & \\
\hline \multicolumn{5}{|l|}{ Ever vaccinated } \\
\hline No & $445(49.6)$ & $39(40.2)$ & $406(50.9)$ & \multirow[t]{2}{*}{0.114} \\
\hline Yes & $589(50.4)$ & $71(59.8)$ & $518(49.1)$ & \\
\hline \multicolumn{5}{|l|}{ Toilet facility } \\
\hline Open defecation & $459(38.0)$ & $51(31.8)$ & $408(38.8)$ & \multirow[t]{3}{*}{0.556} \\
\hline Unimproved facility & $401(53.2)$ & $45(56.1)$ & $356(52.8)$ & \\
\hline Improved facility & $174(8.8)$ & $14(12.1)$ & $160(8.4)$ & \\
\hline \multicolumn{5}{|l|}{ Drinking water source } \\
\hline Improved & $624(54.1)$ & $60(49.7)$ & $564(54.7)$ & \multirow[t]{2}{*}{0.476} \\
\hline Unimproved & $410(45.9)$ & $50(50.3)$ & $360(45.3)$ & \\
\hline \multicolumn{5}{|l|}{ ANC visit } \\
\hline No visit & $336(34.5)$ & $52(49.3)$ & $284(32.4)$ & \multirow[t]{3}{*}{0.049} \\
\hline $1-3$ visits & $341(34.2)$ & $28(22.2)$ & $313(35.9)$ & \\
\hline$\geq 4$ visits & $357(31.3)$ & $30(28.5)$ & $327(31.7)$ & \\
\hline \multicolumn{5}{|l|}{ Place of delivery } \\
\hline Home & $604(63.4)$ & $80(75.7)$ & $524(61.7)$ & \multirow[t]{2}{*}{0.037} \\
\hline Health institution & $430(36.6)$ & $30(24.3)$ & $400(38.3)$ & \\
\hline \multicolumn{5}{|l|}{ Mode of delivery } \\
\hline Vaginal & $996(97.0)$ & $103(95.4)$ & $893(97.3)$ & \multirow[t]{2}{*}{0.368} \\
\hline Cesarean & $38(3.0)$ & $7(4.6)$ & $31(2.7)$ & \\
\hline \multicolumn{5}{|c|}{ Currently breastfeeding } \\
\hline No & $45(4.7)$ & $5(7.0)$ & $40(4.4)$ & \multirow[t]{2}{*}{0.383} \\
\hline Yes & $989(95.3)$ & $105(93.0)$ & $884(95.6)$ & \\
\hline \multicolumn{5}{|c|}{ Time of initiation of breastfeeding } \\
\hline$\leq 1 \mathrm{~h}$ & $716(73.0)$ & $69(70.7)$ & $647(73.4)$ & \multirow[t]{2}{*}{0.715} \\
\hline$>1 \mathrm{~h}$ & $304(27.0)$ & $39(29.3)$ & $265(26.6)$ & \\
\hline \multicolumn{5}{|c|}{ Given anything other than breast milk } \\
\hline No & $858(93.1)$ & $76(88.3)$ & $782(93.8)$ & \multirow[t]{2}{*}{0.068} \\
\hline Yes & $162(6.9)$ & $32(11.7)$ & $130(6.2)$ & \\
\hline \multicolumn{5}{|l|}{ Bottle feeding } \\
\hline No & $920(91.4)$ & $86(76.9)$ & $834(93.4)$ & \multirow[t]{2}{*}{$<0.001$} \\
\hline Yes & $114(8.6)$ & $24(23.1)$ & $90(6.6)$ & \\
\hline
\end{tabular}

Abbreviations: BF Breastfeeding; ANC Antenatal care

infections and fevers [12]. A randomized controlled trial revealed that not breastfeeding was associated with increased risks for childhood morbidities including fever, and gastroenteritis [13]. Additionally, longer duration of breastfeeding complemented with antimalarial drugs was linked with decreased childhood mortality [14].
Moreover, longer breastfeeding duration decreased the odds of recurrent cough [15]. Discontinuation of breastfeeding when the baby had fever/cold has been reported elsewhere [16]. Inadequate nutritionals requirement in early life can result in reduced protection against infections [17]. Because of the greater total immunoglobulin concentrations [18], longer duration and frequent breastfeeding might have important protective effect for infections.

The current study revealed there was a $62 \%$ lower odds of developing cough among exclusively breastfed infants. This finding is consistent with findings from other studies. A longitudinal study conducted in Assam, India revealed low birth weight babies without exclusive breastfeeding were at higher risk for morbidities like acute respiratory tract infections [19]. Similarly, a casecontrol study in Italy revealed that upper and lower respiratory tract infections were strongly associated with lack of breastfeeding [20]. Indeed, lack of breastfeeding was associated with lower respiratory tract infections in a randomized controlled trial [13]. Exclusive breastfeeding decreased the odds of rhinitis in Amazon islands, Brazil [21]. An ordinal increase in breastfeeding duration was associated with decreased risk of recurrent cough in adult life [22]. Moreover, a cohort study in Vietnam revealed exclusive breastfeeding was associated with decreased odds of admission for pneumonia [23]. Similarly, an eight-country cohort finding showed the protective effect of exclusive breastfeeding against respiratory tract infections [24]. In Indonesia, delayed initiation of breastfeeding was associated with increased risks of cough and difficulty in breathing during the first 6 months of life [25]. On the other hand, breastfeeding was not a protective factor for pertussis among unvaccinated infants in Italy [26]. This might be due to the baseline immune capacity of the infants or the nature of the disease against immunologic content of the breast milk. Stakeholders need to design appropriate interventions to help mothers breastfeed their babies in case of childhood illnesses.

In this study, exclusive breastfeeding decreased the odds of diarrhea by $67 \%$. This finding is in line with a study conducted in rural Nepal, where suboptimal breastfeeding practices were associated with higher odds of childhood diarrhea [27]. Early life diarrheal disease among non-breastfed infants was associated with shorten telomere length in adult immune cells [28]. A cross-sectional study conducted in north Gondar zone and Afar region of Ethiopia reported children without breastfeeding/not-exclusive breastfeeding practices were at higher risk for diarrheal diseases [29, 30]. A Demographic and Health Survey (DHS) analysis in nine SubSaharan African countries revealed exclusive breastfeeding was significantly associated with lower risk of 
Table 3 Association between exclusive breastfeeding and diarrhea, illness with fever and illness with cough among infants aged less than 6 months in Ethiopia, EDHS 2016

\begin{tabular}{|c|c|c|c|c|c|c|c|c|c|c|c|c|}
\hline & \multicolumn{4}{|c|}{ Illness with Fever in the last 2 weeks } & \multicolumn{4}{|c|}{ Illness with Cough in the last 2 weeks } & \multicolumn{4}{|c|}{ Diarrhea in the last 2 weeks } \\
\hline & Yes & No & COR $(95 \% \mathrm{Cl})$ & $\operatorname{AOR}^{x}(95 \% \mathrm{Cl})$ & Yes & No & COR $(95 \% \mathrm{CI})$ & $\operatorname{AOR}^{y}(95 \% \mathrm{Cl})$ & Yes & No & COR $(95 \% \mathrm{Cl})$ & $\operatorname{AOR}^{z}(95 \% \mathrm{Cl})$ \\
\hline \multicolumn{13}{|l|}{ EBF } \\
\hline Yes & 92 & 832 & $0.30(0.15,0.59)^{*}$ & $0.34(0.16,0.75)^{*}$ & 148 & 776 & $0.38(0.20,0.72)^{*}$ & $0.38(0.200 .72)^{*}$ & 54 & 870 & $0.23(0.11,0.48)^{*}$ & $0.33(0.13,0.83)^{*}$ \\
\hline No & 30 & 80 & 1.00 & 1.00 & 37 & 73 & 1.00 & 1.00 & 23 & 87 & 1.00 & 1.00 \\
\hline
\end{tabular}

*Statistically significant at $P<0.05$. EBF: Excusive Breastfeeding: COR Crude Odds Ratio; AOR: Adjusted Odds Ratio Cl: Confidence Interval

${ }^{x}$ Adjusted for wealth index, birth order, age at birth, residence, ANC visit, place of delivery, mode of delivery, vaccination, source of drinking water, infant sex and infant age

${ }^{y}$ Adjusted for wealth index, birth order, age at birth, residence, ANC visit, education level, place of delivery, mode of delivery, vaccination, infant sex, source of drinking water and infant age

${ }^{z}$ Adjusted for wealth index, birth order, residence, vaccination, source of drinking water, type of toilet facility and infant age

diarrhea [31]. A similar study using the DHS of Tanzania reported the protective effect of exclusive breastfeeding against diarrheal diseases [32]. Additionally, a cohort study in eight countries revealed the protective effect of exclusive breastfeeding on episodes of diarrhea [24]. Prenatal anxiety which might be related to less exclusive breastfeeding [33] and myths about discontinuation of breastfeeding during diarrheal episodes in some communities [34] should be discouraged [20]. As mothers are more receptive for skill-based interactive demonstrations targeted to exclusive breastfeeding [35], designing novel strategies tailored to exclusive breastfeeding is needed.

This study is not without limitations. First, the crosssectional nature of the study precludes drawing conclusions about the influence of exclusive breastfeeding on childhood illnesses. Second, exclusive breastfeeding and childhood illnesses may be prone to self-reported, recall and social desirability biases. Third, although $24-\mathrm{h}$ recall has been widely used to measure infant feeding practices, a recent study in Ethiopia have shown that a single 24-h recall overestimates exclusive breastfeeding practices [36]. Additionally, this study is prone to selection bias, misclassification and residual confounding. The relatively large sample size, availability of detailed data on confounders, and standardized instruments, highquality data collection are some of the strengths of the current study.

\section{Conclusion}

Exclusive breastfeeding was significantly associated with decreased the odds of illness with fever, illness with cough and diarrhea in a representative, sample of Ethiopian infants. This association remained independent of age of mother at infant's birth, household wealth index, educational level, place of delivery, place of residence, region of residence, antenatal care (ANC checkup), mode of delivery, age of infant, sex of infant, birth order, vaccination status, source of drinking water, wealth index and type of toilet facility. Promoting exclusive breastfeeding throughout the country would have a greater contribution for infant health and development. Hence, in developing countries including Ethiopia, interventions targeting women who had no history of antenatal checkup and home delivery are the most cost effective intervention to strengthen exclusive breastfeeding.

\section{Abbreviations}

ANC: Antenatal Care; AOR: Adjusted Odds ratio; COR: Crude Odds Ratio; DHS: Demographic and Health Survey; EA: Enumeration areas; EBF: Exclusive Breastfeeding; SE: Standard error; VIF: Variance inflation factor; WHO: World Health Organization

\section{Supplementary Information}

The online version contains supplementary material available at https://doi. org/10.1186/s13052-021-01115-3.

\section{Additional file 1.}

\section{Acknowledgments}

The authors are grateful for Woldia University. We also thank Ohio University staff (Haile ZT, Ice G, Johnson L and Lewis M) for the training on complex data analysis.

\section{Authors' contributions}

TM, NBY, BA, ML and MLL conceived and design the study. All authors extracted the data. TM, NBY and MLL analyze the data. BA and ML assisted in the data analysis. TM and NBY drafted the manuscript. All authors read and approve the final manuscript.

Funding

Not applicable

Availability of data and materials

The datasets used for this manuscript are available from the corresponding author upon reasonable request.

\section{Declarations}

Ethics approval and consent to participate

The data were downloaded once approval was obtained from Measure DHS. The original DHS data were collected in confirmation with international and national ethical guidelines. The 2016 EDHS protocol was reviewed and approved by the Federal Democratic Republic of Ethiopia Ministry of Science and Technology and the Institutional Review Board of ICF International.

Consent for publication

Not applicable. 


\section{Competing interests}

The authors declare that they have no competing interests.

\section{Author details}

${ }^{1}$ School of Public Health, College of Health Science, Woldia University, P.o.box: 400, Woldia University, Weldiya, Amhara, Ethiopia. ${ }^{2}$ School of Midwifery, College of Health Science, Woldia University, Weldiya, Amhara, Ethiopia. ${ }^{3}$ Department of Medical Laboratory Science, College of Health Science, Woldia University, Weldiya, Amhara, Ethiopia.

Received: 4 December 2020 Accepted: 14 July 2021 Published online: 03 August 2021

\section{References}

1. Saadeh $\mathrm{R}$, Casanovas C. Implementing and revitalizing the baby-friendly hospital initiative. Food Nutr Bull. 2009;30(2_suppl2):S225-S9.

2. Victora CG, Bahl R, Barros AJ, França GV, Horton S, Krasevec J, et al. Breastfeeding in the 21st century: epidemiology, mechanisms, and lifelong effect. Lancet. 2016;387(10017):475-90. https://doi.org/10.1016/S0140-6736(1 5)01024-7.

3. Haile D, Biadgilign S. Higher breastfeeding performance index is associated with lower risk of illness in infants under six months in Ethiopia. Int Breastfeed J. 2015;10(1):32. https://doi.org/10.1186/s13006-015-0057-2.

4. Central Statistical Agency. The DHS Program ICF. Ethiopian Demographic and Health Survey 2016. Addis Ababa and Rockville: CSA and ICF; 2017.

5. Organization WH, Unicef. Baby-Friendly Hospital Initiative: Revised, updated and expanded for integrated care. 2009.

6. Aborigo RA, Moyer CA, Rominski S, Adongo P, Williams J, Logonia G, et al. Infant nutrition in the first seven days of life in rural northern Ghana. BMC Pregnancy Childbirth. 2012;12(1):76. https://doi.org/10.1186/1471-2393-1276.

7. Horta B, Victora C, Organization WH. Short-term effects of breastfeeding: a systematic review on the benefits of breastfeeding on diarrhoea and pneumonia mortality. Geneva: WHO, apps who int/iris/bitstream/10665/ 95585/1/9789241506120_eng pdf. 2013; 2013.

8. Fetene $\mathrm{N}$, Linnander E, Fekadu B, Alemu H, Omer H, Canavan M, et al. The Ethiopian health extension program and variation in health systems performance: what matters? PLoS One. 2016;11(5):e0156438. https://doi. org/10.1371/journal.pone.0156438.

9. Kim SS, Rawat R, Mwangi EM, Tesfaye R, Abebe Y, Baker J, et al. Exposure to large-scale social and behavior change communication interventions is associated with improvements in infant and young child feeding practices in Ethiopia. PLoS One. 2016;11(10):e0164800. https://doi.org/10.1371/journal. pone.0164800

10. Croft NT, AMJ M, Allen CK, et al. Guide to DHS statistics. Rockville: ICF; 2018. p. 2018.

11. Biks GA, Berhane Y, Worku A, Gete YK. Exclusive breast feeding is the strongest predictor of infant survival in Northwest Ethiopia: a longitudinal study. Journal of health, population, and nutrition. 2015;34:9.

12. Ajetunmobi OM, Whyte B, Chalmers J, Tappin DM, Wolfson L, Fleming M, et al. Breastfeeding is associated with reduced childhood hospitalization: evidence from a Scottish Birth Cohort (1997-2009). J Pediatr. 2015;166(3): 620-5.e4.

13. Bork KA, Cournil A, Read JS, Newell ML, Cames C, Meda N, et al. Morbidity in relation to feeding mode in African HIV-exposed, uninfected infants during the first 6 mo of life: the Kesho bora study. Am J Clin Nutr. 2014; 100(6):1559-68. https://doi.org/10.3945/ajcn.113.082149.

14. K'Oyugi BO. Effects of bed nets and anti-malaria drugs use on childhood mortality in Kenya's malaria endemic and epidemic areas. BMC Public Health. 2015;15(1):34. https://doi.org/10.1186/s12889-015-1398-x.

15. Lodge CJ, Dharmage SC. Breastfeeding and perinatal exposure, and the risk of asthma and allergies. Curr Opin Allergy Clin Immunol. 2016;16(3):231-6. https://doi.org/10.1097/ACl.0000000000000266.

16. Kamath SP, Garg D, Khan MK, Jain A. Perceptions and Practices regarding Breastfeeding among Postnatal Women at a District Tertiary Referral Government Hospital in Southern India, vol. 2016; 2016. p. 5430164.

17. Karim T, Muhit M, Khandaker G. Interventions to prevent respiratory diseases - nutrition and the developing world. J Obstet Gynaecol India. 2017;22:31-7.

18. Pedersen SH, Wilkinson AL, Andreasen A, Kinung'hi SM, Urassa M, Michael $D$, et al. Longitudinal analysis of mature breastmilk and serum immune composition among mixed HIV-status mothers and their infants. Clin Nutr (Edinburgh, Scotland). 2016:35(4):871-9.

19. Borah M, Baruah R. Morbidity status of low birth weight babies in rural areas of Assam: a prospective longitudinal study. J Fam Med Prim Care. 2015:4(3): 380-3. https://doi.org/10.4103/2249-4863.161326.

20. Martines F, Salvago P, Ferrara S, Messina G, Mucia M, Plescia F, et al. Factors influencing the development of otitis media among Sicilian children affected by upper respiratory tract infections. Brazil J Otorhinolaryngol. 2016; 82(2):215-22. https://doi.org/10.1016/j.bjorl.2015.04.002

21. Freitas MS, de Cordoba LF, Monteiro JC, Sole D. Prevalence of rhinitis and associated factors in schoolchildren who live in the Amazon islands. Am J Rhinol Allergy. 2016;30(3):79-82. https://doi.org/10.2500/ajra.2016.30.4293.

22. Gerhart KD, Stern DA, Guerra S, Morgan WJ, Martinez FD, Wright AL. Protective effect of breastfeeding on recurrent cough in adulthood. Thorax. 2018:73(9):833-9. https://doi.org/10.1136/thoraxinl-2017-210841.

23. Hanieh S, Ha TT, Simpson JA, Thuy TT, Khuong NC, Thoang DD, et al. Exclusive breast feeding in early infancy reduces the risk of inpatient admission for diarrhea and suspected pneumonia in rural Vietnam: a prospective cohort study. BMC Public Health. 2015;15(1):1166. https://doi. org/10.1186/s12889-015-2431-9.

24. Richard SA, McCormick BJJ, Seidman JC, Rasmussen Z, Kosek MN, Rogawski ET, et al. Relationships among common illness symptoms and the protective effect of breastfeeding in early childhood in MAL-ED: an eightcountry cohort study. Am J Trop Med Hygiene. 2018;98(3):904-12. https:// doi.org/10.4269/ajtmh.17-0457.

25. Lee KJ, Bines JE, Watts E, Satria CD, Atthobari J, Nirwati H, et al. Delayed Breastfeeding Initiation Is Associated with Infant Morbidity. BMC Pediatr 2017:191:57-62.e2

26. Pandolfi E, Gesualdo F, Carloni E, Villani A, Midulla F, Carsetti R, et al. Does breastfeeding protect young infants from pertussis? Case-control study and immunologic evaluation. Pediatr Infect Dis J. 2017;36(3):e48-53. https://doi. org/10.1097/INF.0000000000001418.

27. Acharya D, Singh JK, Adhikari M, Gautam S, Pandey P, Dayal V. Association of water handling and child feeding practice with childhood diarrhoea in rural community of southern Nepal. J Infect Public Health. 2018;11(1):69-74. https://doi.org/10.1016/j.jiph.2017.04.007.

28. Eisenberg DTA, Borja JB, Hayes MG, Kuzawa CW. Early life infection, but not breastfeeding, predicts adult blood telomere lengths in the Philippines. Am J Hum Biol. 2017;29(4).

29. Getachew A, Guadu T, Tadie A, Gizaw Z, Gebrehiwot M, Cherkos DH. Diarrhea prevalence and sociodemographic factors among under-five children in rural areas of North Gondar zone, Northwest Ethiopia, vol. 2018; 2018. p. 6031594.

30. Gizaw Z, Woldu W, Bitew BD. Child feeding practices and diarrheal disease among children less than two years of age of the nomadic people in Hadaleala District, Afar region, Northeast Ethiopia. Int Breastfeed J. 2017; 12(1):24. https://doi.org/10.1186/s13006-017-0115-z.

31. Ogbo FA, Agho K, Ogeleka P, Woolfenden S, Page A, Eastwood J. Infant feeding practices and diarrhoea in sub-Saharan African countries with high diarrhoea mortality. PLoS One. 2017;12(2):e0171792. https://doi.org/10.1371/ journal.pone.0171792.

32. Ogbo FA, Nguyen $\mathrm{H}, \mathrm{Naz} \mathrm{S}$, Agho KE, Page A. The association between infant and young child feeding practices and diarrhoea in Tanzanian children. Trop Med Health. 2018;46(1):2. https://doi.org/10.1186/s41182-018-0084-y.

33. Field T. Prenatal anxiety effects: a review. Infant Behav Develop. 2017:49: 120-8. https://doi.org/10.1016/j.infbeh.2017.08.008.

34. Etokidem AJ, Johnson O. Child survival strategies: assessment of knowledge and practice of rural women of reproductive age in Cross River state, Nigeria. J Trop Med. 2016;2016:5098463.

35. Greenland K, Chipungu J, Chilekwa J, Chilengi R, Curtis V. Disentangling the effects of a multiple behaviour change intervention for diarrhoea control in Zambia: a theory-based process evaluation. Glob Health. 2017;13(1):78 https://doi.org/10.1186/s12992-017-0302-0.

36. Fenta EH, Yirgu R, Shikur B, Gebreyesus SH. A single $24 \mathrm{~h}$ recall overestimates exclusive breastfeeding practices among infants aged less than six months in rural Ethiopia. Int Breastfeed J. 2017;12(1):36. https://doi. org/10.1186/s13006-017-0126-9.

\section{Publisher's Note}

Springer Nature remains neutral with regard to jurisdictional claims in published maps and institutional affiliations. 